\title{
Selective Detection of Specific Protein-Ligand Complexes by Electrosonic Spray-Precursor Ion Scan Tandem Mass Spectrometry
}

\author{
Noemi Czuczy, Maria Katona, and Zoltan Takats \\ Cell Screen Applied Research Center, Semmelweis University, Budapest, Hungary
}

\begin{abstract}
A novel mass spectrometric method for the selective detection of specific protein-ligand complexes is presented. The new method is based on electrosonic spray ionization of samples containing protein and ligand molecules, and mass spectrometric detection using the precursor ion scanning function on a triple quadrupole instrument. Mass-selected intact proteinligand complex ions are subjected to fragmentation by means of collision-induced dissociation in the collision cell of the instrument, while the second mass analyzer is set to the $\mathrm{m} / \mathrm{z}$ of protonated ligand ions or their alkali metal adducts. The method allows for the detection of only those ions which yield ions characteristic of the ligand molecules upon fragmentation. Since the scan range of first analyzer is set well above the $\mathrm{m} / \mathrm{z}$ of the ligand ion, and the CID conditions are established to permit fragmentation of only loosely bound, noncovalent complexes, the method is specific to the detection of protein-ligand complexes under described conditions. Behavior of biologically specific and nonspecific complexes was compared under various instrumental settings. Parameters were optimized to obtain maximal selectivity for specific complexes. Specific and nonspecific complexes were found to show markedly different fragmentation characteristics, which can be a basis for selective detection of complexes with biological relevance. Preparation of specific and nonspecific complexes containing identical building blocks was attempted. Complex ions with identical stoichiometry but different origin showed the expected difference in fragmentation characteristics, which gives direct evidence for the different mechanism of specific versus nonspecific complex ion formation. (J Am Soc Mass Spectrom 2009, 20, 227-237) () 2009 Published by Elsevier Inc. on behalf of American Society for Mass Spectrometry
\end{abstract}

$\mathrm{M}$ ass spectrometric investigation of noncovalent complexes has been an issue of scientific interest since the appearance of soft ionization methods in the 1970s. Originally mass spectrometric studies of these complexes were aimed at the determination of thermodynamic constants, such as proton affinity, and for the fundamental understanding of gas-phase ion molecule reactions [1]. The advent of methods capable of ionizing macromolecular species has changed the focus of this subfield towards the investigation of biologically relevant, macromolecular complexes [2, 3]. Since the early 1990s, numerous methods have been developed for the investigation proteinligand, multiprotein, and nucleic acid complexes, mainly applying electrospray-based methods [4-6]. Matrix-assisted laser desorption/ionization (MALDI) and other laser desorption/ionization methods were also shown to be capable of producing protein-protein complex ions [7, 8]. MALDI investigation of noncovalent complexes using covalent cross-linking of the substrate has been shown to be a useful and widely

Address reprint requests to Dr. Z. Takats, Cell Screen Applied Research Center, Semmelweis University, Ulloi ut 93, Budapest, Hungary. E-mail: takats@gyer1.sote.hu applicable technique, especially for the localization of the binding site [9]. Investigation of intact complexes by MALDI has also been accomplished utilizing the socalled "first-shot" phenomenon [10]. This phenomenon is observed when the first laser shot produces a MALDI-MS spectrum featuring protein-ligand complexes on freshly prepared sample spots. The mechanism of the first-shot phenomenon was associated with segregation of folded protein-ligand complexes on the surface of sample spots. Although MALDI-MS methods employing traditional matrices lack physiological conditions during sample preparation (especially due to acidity of these compounds), application of nonacidic matrices has been shown to overcome this problem [11]. The recent development of matrix-free laser desorption/ionization (LDI) methods and MALDI methods employing a Nd-YAG laser and water as matrix have the potential of extending the application of LDI methods to the investigation of noncovalent complexes [12].

Mass spectrometric investigation of noncovalent complexes of biologically relevant macromolecules can provide information on the stoichiometry and relative stability of these complexes [13]. The behavior of proteinligand complexes carries extreme importance from a
(C) 2009 Published by Elsevier Inc. on behalf of American Society for Mass Spectrometry. 1044-0305/09/\$32.00

doi:10.1016/j.jasms.2008.09.010
Published online September 18, 2008 Received May 25, 2008 Revised September 6, 2008 Accepted September 8, 2008 
pharmacology point of view, since the general mechanism of drug action starts with the binding of a drug molecule to its target protein (or nucleic acid). Hence, in the early stage of drug development, an enormous number of binding assays are performed, either to find the best drug candidates from a family of similar molecules, or to find a protein target for molecules with known biological effects [14]. General approaches to test protein-ligand interactions include affinity chromatography, ultrafiltration, dialysis, and a number of other solution phase methods [15]. Mass spectrometry carries multiple advantageous features compared with these methods, including high sensitivity and specificity. High sensitivity of mass spectrometric methods results in considerably lower sample consumption, while specificity enables parallel testing of multiple species and lowers purity requirements on the samples. Nevertheless, in spite of these advantages, mass spectrometric methods share a common, intrinsic disadvantage of being gas-phase methods, detecting separated gaseous species. In the case of complex formation equilibrium studies, this feature is critical. In the most widely utilized electrospray technique, changes in the solution phase concentration of the studied species caused by solvent evaporation from sprayed droplets may occur. Furthermore, the reversible complex formation process becomes virtually unidirectional in the atmospheric interface and vacuum regime of mass spectrometer. Although the concentration process and dissociation of complex ions in the gas phase act in opposite directions, the resulting ion abundance ratios have no straightforward relationship to the original, solution phase ratios of concentrations. Further limitations of mass spectrometric investigation of protein-ligand complexes include different response factors of the mass spectrometer to different species and the strict restrictions on appropriate sample composition for electrospray ionization (e.g., absence of nonvolatile salts). There has been ongoing research aimed at developing ionization methods and atmospheric interface setups, which minimize the above listed disadvantageous features [16-18]. Other studies were aimed at establishing a clear relationship between mass spectrometric data and stability constants determined in solution phase $[19,20]$.

Electrospray ionization of macromolecular complexes starts with the formation of multiply charged droplets from a bulk solution phase [21]. Multiply charged droplets lose solvent by evaporation, in effect the droplets reach Rayleigh instability limit with regard to their charge-to-volume ratio. When droplets reach instability, they either divide into smaller droplets or directly emit low molecular weight $(\mathrm{MW}<1000)$ gaseous ions. In the case of macromolecular species, final ion formation follows the so called "charged residue model" [21]. The model assumes that final droplets that contain a single macromolecule lose all their water content, yielding a fully desolvated gaseous ion. Nascent ions traveling through the atmospheric interface of the mass spectrometer (conducted by neutral gas flow and electrostatic potential gradient) undergo multiple collisions, which result in elevated internal energy, and may eventually lead to dissociation/fragmentation of the ions.

During the above-described process, there is a clear increase in the concentration of all nonvolatile species as the solvent is evaporated from the electrosprayed droplets. To eliminate the shift of complex formation equilibrium, the size of the initial droplets needs to be minimized while the ion formation process has to be accelerated. Acceleration of gas-phase ion formation is also preferred for better ion survival. It was shown [17, 18] that if the desolvation of ions takes place in the atmospheric interface of the mass spectrometer, milder desolvation conditions (atmospheric interface temperature, cone voltage) can be employed, yielding a higher survival rate of delicate species. This can be achieved by choosing an appropriate ionization method like nanospray and electrosonic spray ionization. Minimized shift in solution phase equilibria during electrosonic spray ionization (ESSI) ionization has been demonstrated recently [20]. Survival of complexes in the atmospheric interface and ion optics can be further improved by elevating the pressure in the high vacuum regime of instruments [16]. This latter method is based on the collisional cooling of species which had been heated in the atmospheric interface.

The investigation of the relationship between solution phase concentration ratios and mass spectrometric ion abundance ratios has also been an issue of scientific interest. Recently, a novel approach was developed for the mathematical treatment of mass spectrometric data, which yields stability constants highly similar to those determined by solution phase techniques [22]. The combination of ESSI and a novel data interpretation method was demonstrated to be successful for the determination of stability constants relevant to solution phase systems [20].

Various applications of mass spectrometric detection of protein-ligand complexes have been demonstrated, including high-throughput testing of combinatorial libraries on protein targets and determining relative stability constants of complexes, among many others. Certain applications, such as collision induced dissociation in FTICR-MS were found useful for determination of exact mass and relative abundance of ligands. Tandem mass spectrometry allows for further characterization of ligands, e.g., determination of molecular structure or differentiation of isomers [23]. Although these applications are all promising [24-26], their practical application is still not widespread. Drug development studies still employ mass spectrometry as a detection tool for proteins separated by affinity chromatography, or for determination of amounts of ligand bound by a protein target [14, 27, 28].

The present paper demonstrates a novel approach for the analysis of protein-ligand complexes, an approach which, after further development, can poten- 
tially be the basis for a mass spectrometric target screening method. The new method yields mass spectrometric response factors for complexes of a single ligand with various proteins being proportional to the known stability constants of the complexes in solution. This feature is achieved by electrosonic spray ionization of protein mixtures containing the ligand molecule and performing a precursor ion scan for the molecular ion of the ligand. The method is shown to be capable of differentiating specific complexes formed in solution phase from nonspecific complexes formed in the atmospheric interface of the mass spectrometer. Proof-ofprinciple is demonstrated in the present work and the method is characterized using model systems.

\section{Experimental}

Ribonuclease A (from bovine pancreas), lysozyme (from chicken egg white purity: $\sim 95 \%$ ), cytosine (purity: $99 \%)$, and maltotriose ( $\geq 96 \%)$ were purchased from Sigma (Sigma-Aldrich Inc., St. Louis, MO). Cytochrome $c$ (from equine heart) was obtained from Calbiochem (Merck Chemical Ltd., Nottingham, UK) (purity: 95.9\%). All compounds except ribonuclease A were used without further purification. Purification of ribonuclease A was performed on Microcon YM-10 centrifugal filter devices (Millipore Corp., Billerica, MA). The protein was washed 3 times with $450 \mu$ l ammoniumacetate ( $\mathrm{pH}$ 6.08) buffer. Raw ribonuclease fraction was obtained from bovine pancreatic secretion following the method of Hirs et al. [29]. Stock solutions were prepared in ammonium acetate buffer; initial concentration was $1 \mathrm{mg} / \mathrm{ml}$ for both proteins and ligands.

Spectra were acquired on an Applied Biosystems / MDS SCIEX 4000QTRAP instrument (MDS Inc., Concord, Ontario) equipped with a home-built ESSI ionsource (Figure 1a). A Swagelok stainless steel T-element was used for the spray housing. Detailed architecture of the sprayer was described previously [17]. Sample was introduced through a fused silica capillary (i.d. 50, o.d. 150), and nebulizing gas flowed through $1 / 16$-in. stainless steel tubing. Fused silica capillary with internal diameter and o.d. of 255 and $360 \mu \mathrm{m}$, respectively, was used as the nebulizing gas outlet. The experimental setup is shown in Figure 1b. High voltage for the electrosonic spray was set to $3000 \mathrm{~V}$. Nebulizing gas inlet pressure was varied from 1 to 10 bar. Sample was introduced by a Harvard Apparatus (Holliston, MA) PHD 2000 syringe pump (flow rate $1 \mu \mathrm{L} / \mathrm{min}$ ). Spectra were acquired at different interface heater temperatures $\left(40-200{ }^{\circ} \mathrm{C}\right)$ and declustering potentials (50-350 $\mathrm{V})$. Data for systematic characterization of the method was acquired in SIM or MRM mode, in five replicates for each data point.

Dual spray experiment was carried out using two identical ESSI sprayers [17] in a geometrical setup depicted in Figure 1c. One sprayer was operated in ESSI mode, while the other in SSI mode, i.e., without high voltage applied. Buffered aqueous solution of the pro- tein was sprayed in the ESSI source, while a solution of ligand was sprayed orthogonally in sonic spray mode. A grounded separating plate was placed between sprays (see Figure 1a and c) to minimize negative ion formation in the sonic spray source. Intercepting sprays produce complexes of the two sprayed species.

\section{Results and Discussion}

The electrosonic spray mass spectrum and corresponding precursor ion mass spectrum of bovine pancreatic lysate spiked with cytosine are shown in Figure $2 \mathrm{a}$ and b, respectively. While Figure $2 \mathrm{a}$ shows poorly resolved peaks, Figure $2 b$ shows two well defined multiply charged species corresponding to the bovine RNase A-cytosine complex. Since a triple quadrupole mass spectrometer in precursor ion scan mode for the protonated molecular ion of cytosine $(\mathrm{m} / \mathrm{z} 179)$ detects only those species which produce this ion upon CID, this scan function can be utilized for the selective detection of cytosine binding species. Another example is of hen egg-white lysozyme and maltotriose shown in Figure 2c and $2 \mathrm{~d}$. Similar to the case described above, recording the precursor ion scan for the potassium adduct ion of maltotriose $(m / z 543)$ yields a mass spectrum featuring mainly the multiply charged ions of those molecules that strongly bind maltotriose, i.e., lysozyme in this case.

As it is shown in Figure 2, the precursor ion scan method for the molecular ion of a component of a mixture yields mass spectra, which feature only those components that bind the corresponding molecule, and their relative peak intensities (versus their intensities in single stage MS) reflect their relative affinity to the ligand molecule. The general scheme of the method is shown in Figure 3. The method is based on the previous observation that specific protein-ligand complexes survive spray ionization, and certain varieties of electrospray ionization do not yield large amounts of artifactual complexes. These varieties of electrospray include nanospray [30, 31] and electrosonic spray [17]. A further working condition for the method is that the proteinligand complex dissociates by CID to produce ligand molecule in ionic form.

When a ligand molecule of interest is mixed with a mixture of protein molecules that potentially bind the ligand, solvent phase equilibrium follows the individual binding constants of the potentially formed complexes (Figure 3). The mixture then undergoes electrospray ionization and the ion population enters the mass spectrometer, while a large proportion of complex ions dissociate. The remaining ions are mass selected in the first quadrupole analyzer and then dissociate in the collision cell to yield protein and ligand ions. Because the second quadrupole analyzer is set to the $m / z$ of the ligand (protonated or alkali metal adduct) molecular ion (Figure 3), a signal is only detected when the fragmentation of complexes yields ligand ions. This condition is fulfilled in the case of molecular com- 


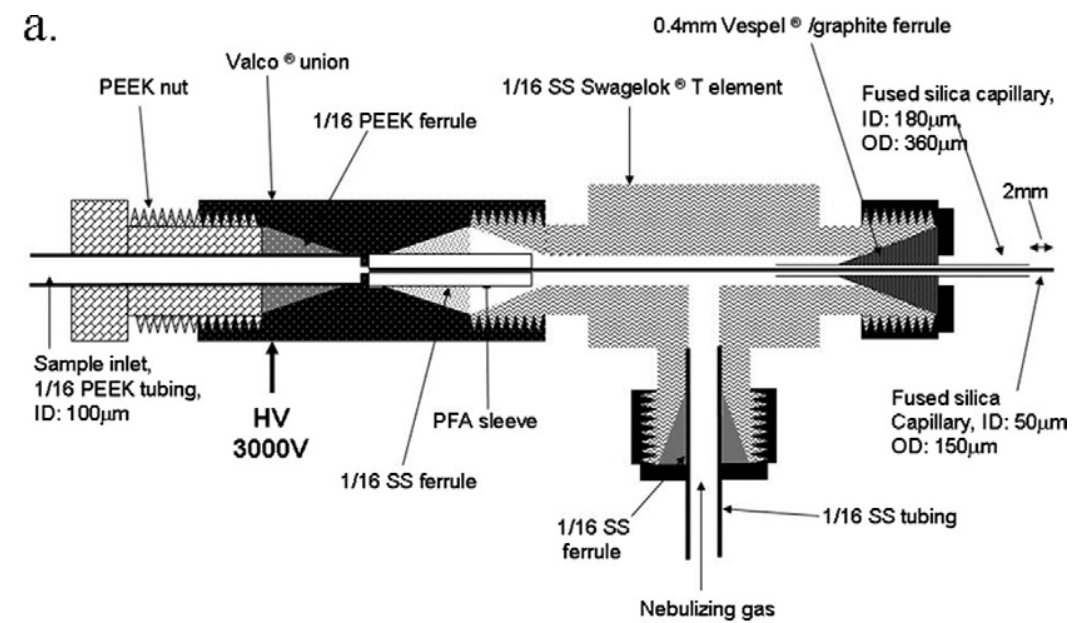

b.

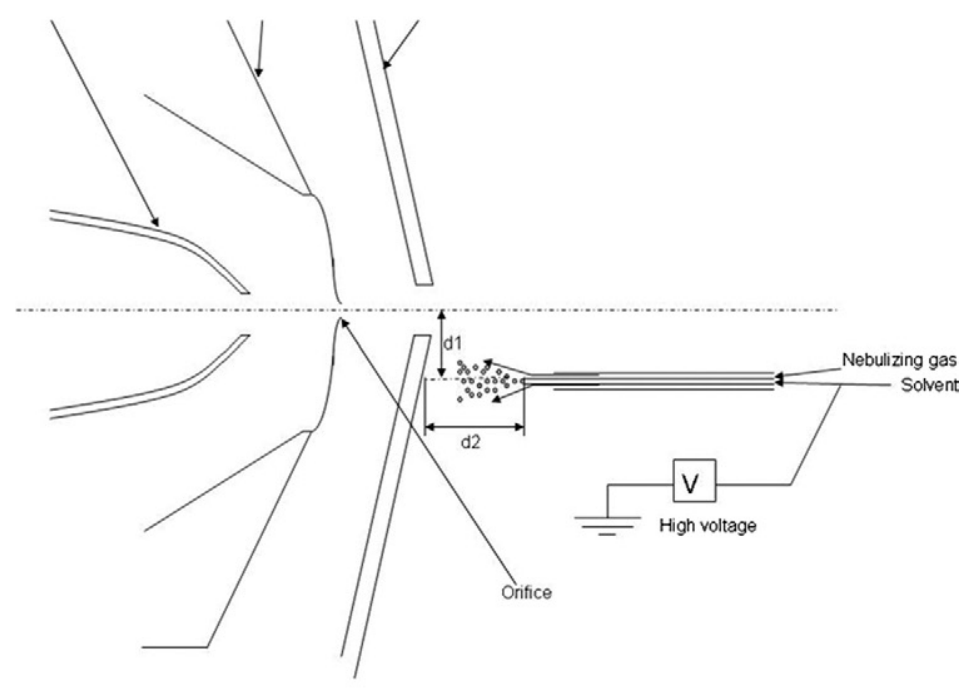

c.

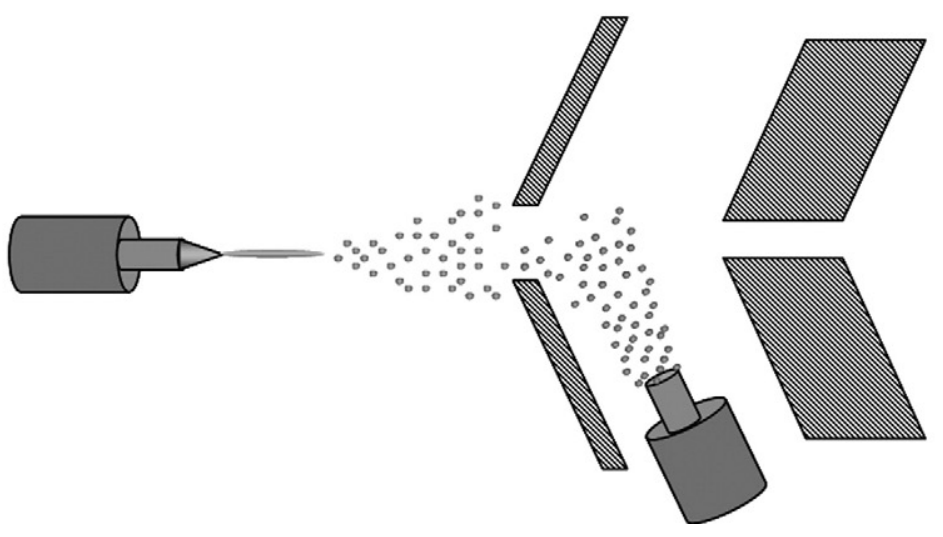

Figure 1. (a) Scheme of the in-house ESSI source (b) Experimental setup of ESSI experiments. (c) Experimental setup of dual-spray experiments. Sprayer facing atmospheric interface was used in ESSI mode to generate multiply charged protein ions, while other sprayer was used in SSI mode to generate mostly neutral aerosol of the ligand.

plexes, which contain the ligand molecule and where relative proton (or alkali metal) affinities of fragments allow the formation of ionized ligand molecule upon fragmentation.

To maximize the performance of the method with regard to specificity and sensitivity, the following the- oretical considerations have to be taken into account: (1) relatively low ligand concentrations are preferred because when all potential binding sites are saturated in solvent phase, the resulting data will reflect the relative dissociation kinetics; (2) ion-source and atmospheric interface parameters have to be optimized to minimize 


\section{a.}

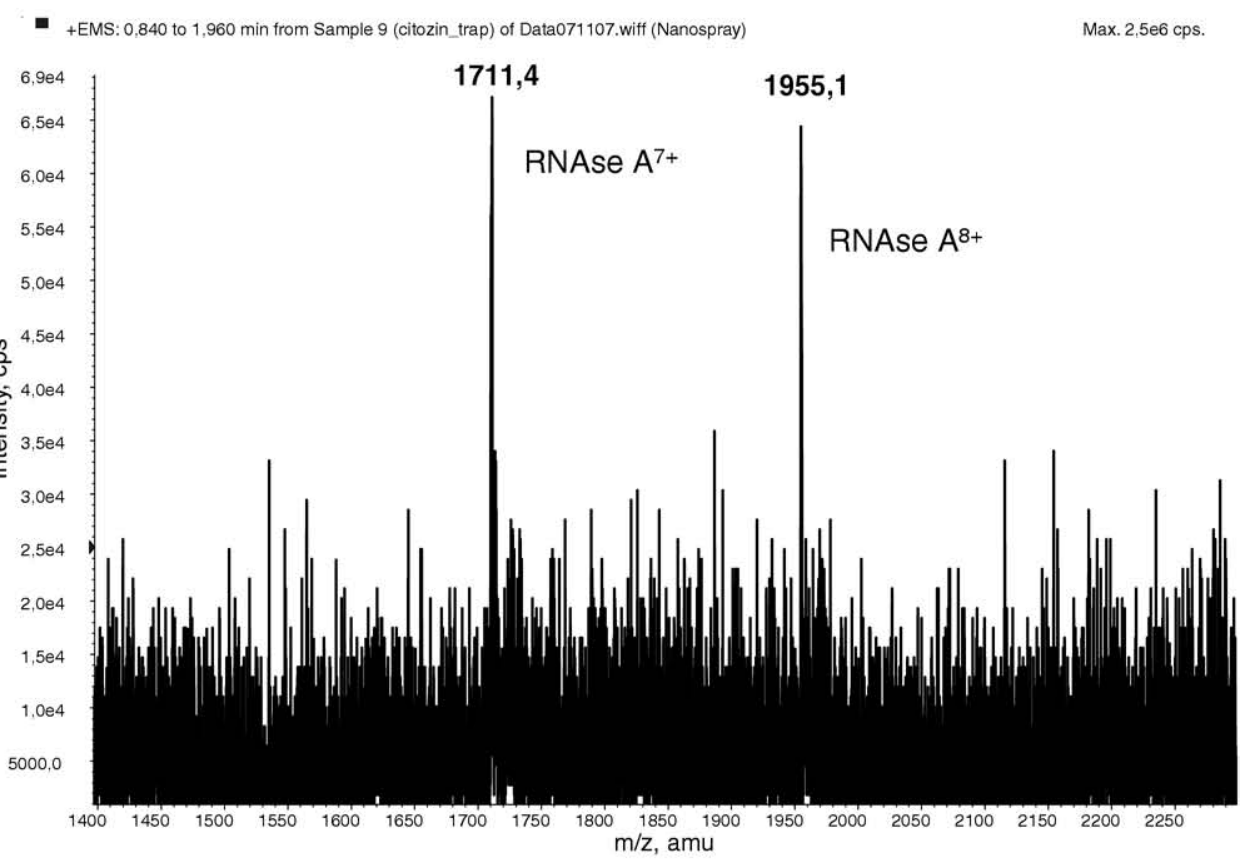

b.

- +Prec (112,00): 0,835 to 2,072 min from Sample 4 (citozin_prec_dp90) of Data071107.wiff (Nanospray) Max. 3421,2 cps.

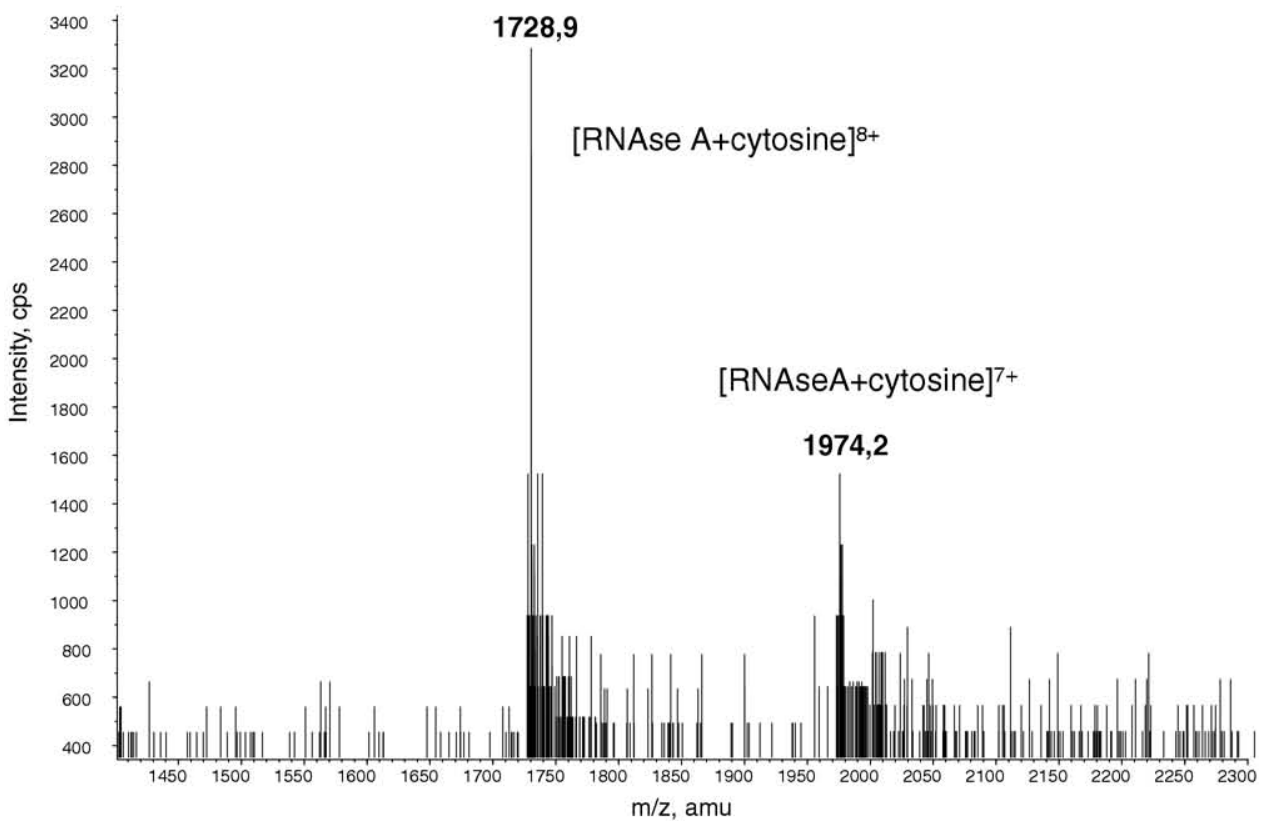

Figure 2. (a) Single stage electrosonic spray mass spectrum of bovine pancreatic protein fraction spiked with cytosine. (b) Cytosine precursor ion mass spectrum of bovine pancreatic protein fraction spiked with cytosine. (c) Single stage electrosonic spray mass spectrum of hen egg-white lysozyme, cytochrome $c$, and maltotriose. (d) Maltotriose precursor ion mass spectrum of hen egg-white lysozyme, cytochrome $c$, and maltotriose.

the shift of complex formation equilibrium in solvent phase and to minimize complex dissociation in the gas-phase following ion formation and during ion transfer; (3) CID parameters have to be chosen to maximize the yield of ligand ions upon fragmentation of complexes. It is important to note that higher charge state complex ions are more likely to produce a charged ligand fragment, presumably due to the effect of Coulomb-repulsion on effective site-specific proton affinities of the proteins [32].

The experiment described above has a number of potential application areas, including identification of 


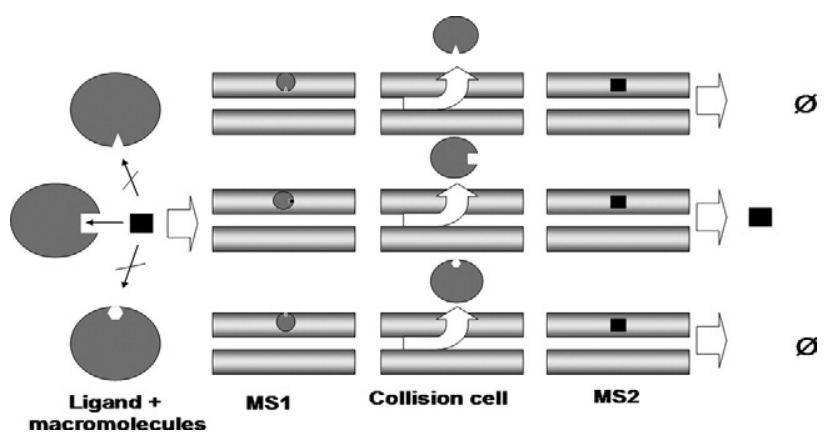

Figure 3. Scheme of target finding. The ligand of interest (represented by a square) is mixed with proteins (circles), and specific complexes are formed. The mass spectrometer is operated in the precursor ion scan mode. Pair wise rectangles represent the quadrupoles rods, small circles and rectangles on the rods are the molecules that can get through the given quadrupole. Signal is detected only at the masses of protein-ligand complexes.

target proteins for drug candidate molecules, tracing molecular interactions in fundamental biochemistry, and understanding the ionization mechanism of protein-ligand complexes.

RNAse A-cytosine and lysozyme-maltotriose complexes were used as model systems for systematic characterization of the method. Cytochrome $c$ was used in both cases as the reference protein, which presumably does not show any specific binding for the chosen ligands. Various potential ligands, including substrates and inhibitors, were tested for both enzymes. Model systems are listed in Table 1. In case of certain substrates, enzymatic reaction has taken place on fragmentation of the complex (e.g., tetra- $N$-acetyl chitotetraose), and precursor ion scan was only successfully performed for the molecular ion of the product of enzymatic reaction (e.g., di- $\mathrm{N}$-acetyl chitobiose).

Instrumental parameters that were tested are listed in Table 2, together with the tested range and magnitude of observed effects. As shown in Table 2, while solution temperature, atmospheric interface temperature, declustering potential, and nebulizing gas flow rate showed dramatic influences on the observed signal in both single stage MS and precursor ion mode, the remaining parameters did not show remarkable effects.

Solution temperature dependence of single stage MS signal and precursor ion signal of studied complexes is shown in Figure $4 a$ and $b$, respectively. This parameter was tested to obtain information on the origin of the observed ions. Complex formation equilibria are almost always shifted towards dissociation with elevated temperatures due to the entropy factor of dissociative/ associative reactions. The assumption was that a shift in solution phase equilibrium is observed if the detected complex ions are solution-originated. As Figure 4a and b show, the expected shift both in the ratio of free protein and complex ions and absolute intensity of complex ions was clearly observed. Since changing the temperature of the sample does not cause significant change in the temperature of the spray, it can be concluded that solution phase equilibrium has a strong effect on the experimental results. There are two explanations for the limited dependence of spray temperature on sample temperature: (1) the relative mass flow of the liquid sample and nebulizing gas, and (2) the experimental setup that employs a fairly distant heated sample reservoir. According to mass flow of aqueous sample and nitrogen gas, a change of $10^{\circ} \mathrm{C}$ in the temperature of the sample results in less than $1^{\circ} \mathrm{C}$ change in the temperature of the spray. Taking the results of ion-source temperature dependence (shown in Figure $4 \mathrm{c}$ and $\mathrm{d}$ ) into account, the slight alteration of spray temperature as the result of varying solvent temperature in itself does not yield measurable effect. The effect of solution temperature has only a poorly definable effect on the dissociation of nonspecific complexes (Figure $4 \mathrm{a}$ and $\mathrm{b}$ ). This latter observation can be tenta-

Table 1. Tested protein-ligand systems

\begin{tabular}{|c|c|c|c|c|c|c|}
\hline \multirow[b]{3}{*}{ Protein } & \multirow[b]{3}{*}{ Ligand } & \multirow{3}{*}{$\begin{array}{l}\text { Relative intensity } \\
\text { (1:1 stoichiometry } \\
\text { complex/protein) }\end{array}$} & \multicolumn{4}{|c|}{ Precursor scan } \\
\hline & & & \multicolumn{2}{|c|}{$[\mathrm{M}+\mathrm{H}]^{+}$} & \multicolumn{2}{|c|}{$[\mathrm{M}+\mathrm{Na}]^{+} /[\mathrm{M}+\mathrm{K}]^{+}$} \\
\hline & & & $+1-$ & $m / z$ & $+1-$ & $m / z$ \\
\hline \multirow[t]{7}{*}{ RNase A } & adenine & 0.2 & - & 136 & + & $158 / 175$ \\
\hline & adenosine & - & - & 268 & $(+)$ & $290 / 307$ \\
\hline & adenylyl $\left(3^{\prime}-5^{\prime}\right)$ cytidine & 0.4 & - & 573 & + & $595 / 612$ \\
\hline & cytosine & 0.8 & + & 112 & - & $134 / 151$ \\
\hline & cytidylyl(3'-5')uridine & 0.9 & - & 550 & $+^{\mathrm{a}}$ & $572 / 589$ \\
\hline & guanylyl $\left(3^{\prime}-5^{\prime}\right)$ uridine & 1.7 & - & 589 & $++^{a}$ & $611 / 628$ \\
\hline & uridylyl $\left(3^{\prime}-5^{\prime}\right)$ uridine & 0.2 & - & 550 & $(+)$ & $572 / 589$ \\
\hline \multirow[t]{5}{*}{ Lysozyme } & maltose & 4.9 & - & 343 & + & $365 / 404$ \\
\hline & chitotriose & 0.4 & ++ & 629 & - & $651 / 668$ \\
\hline & maltotriose & 0.3 & - & 505 & ++ & $527 / 544$ \\
\hline & hexa- $N$-acetylchitohexaose & 1.2 & - & 1238 & $+^{a}$ & $1260 / 1277$ \\
\hline & tetra- $N$-acetylchitotetraose & 1.6 & - & 832 & $+{ }^{a}$ & $854 / 871$ \\
\hline
\end{tabular}

${ }^{a}$ Enzymatic reaction takes place under experimental conditions, precursor ion scan was successfully performed for product. 
Table 2. Tested instrumental parameters and their optimal ranges

\begin{tabular}{lllc}
\multicolumn{1}{c}{ Parameter } & Range tested & Optimal value & $\begin{array}{c}\text { Observed variation in range } \\
\text { (order of magnitude) }\end{array}$ \\
\hline \hline Solution temperature & ambient $-90^{\circ} \mathrm{C}$ & $40{ }^{\circ} \mathrm{C}$ & $3-4$ \\
Atmospheric interface temperature & ambient $-250^{\circ} \mathrm{C}$ & $70-120{ }^{\circ} \mathrm{C}$ & $1-2$ \\
Declustering potential & $50-200 \mathrm{~V}$ & $120-170 \mathrm{~V}$ & $1-2$ \\
Collision energy & $5-35 \mathrm{eV} / \mathrm{unit}$ charge & $15 \mathrm{eV} / \mathrm{unit}$ charge & $<1$ \\
Spray HV & $1500-5500 \mathrm{~V}$ & $4500 \mathrm{~V}$ & $<1$ \\
Nebulizing gas nominal linear velocity & $0-400 \mathrm{~m} / \mathrm{s}$ & $350-400 \mathrm{~m} / \mathrm{s}$ & $1-2$ \\
Sample flow rate & $0.03-3 \mu \mathrm{L} / \mathrm{min}$ & $1 \mu \mathrm{L} / \mathrm{min}$ & $<1$ \\
Sprayer-to-instrument distance & $3-50 \mathrm{~mm}$ & $15 \mathrm{~mm}$ & $<1$ \\
\hline
\end{tabular}

tively associated with the fact that nonspecific complexes do not have a well-defined structure, i.e., there are a number of potential binding sites on any protein, yielding a number of different complex structures. However, the non-zero abundance of these complexes under arbitrary conditions indicates that complexes are formed not only in the bulk solution phase, but there is also minor complex formation in the spray and the atmospheric interface (vide infra: results of dual spray experiments). Other solution phase effects, including $\mathrm{pH}$ of the solution sample, protein concentration, and presence of organic solvents have also yielded the expected shifts in protein/complex abundance ratios (data not shown).

The effect of the spraying parameters was in agreement with previous observations. Spray geometry has a general effect on ion abundances without dramatically changing complex/protein abundance ratios. In contrast, gas flow
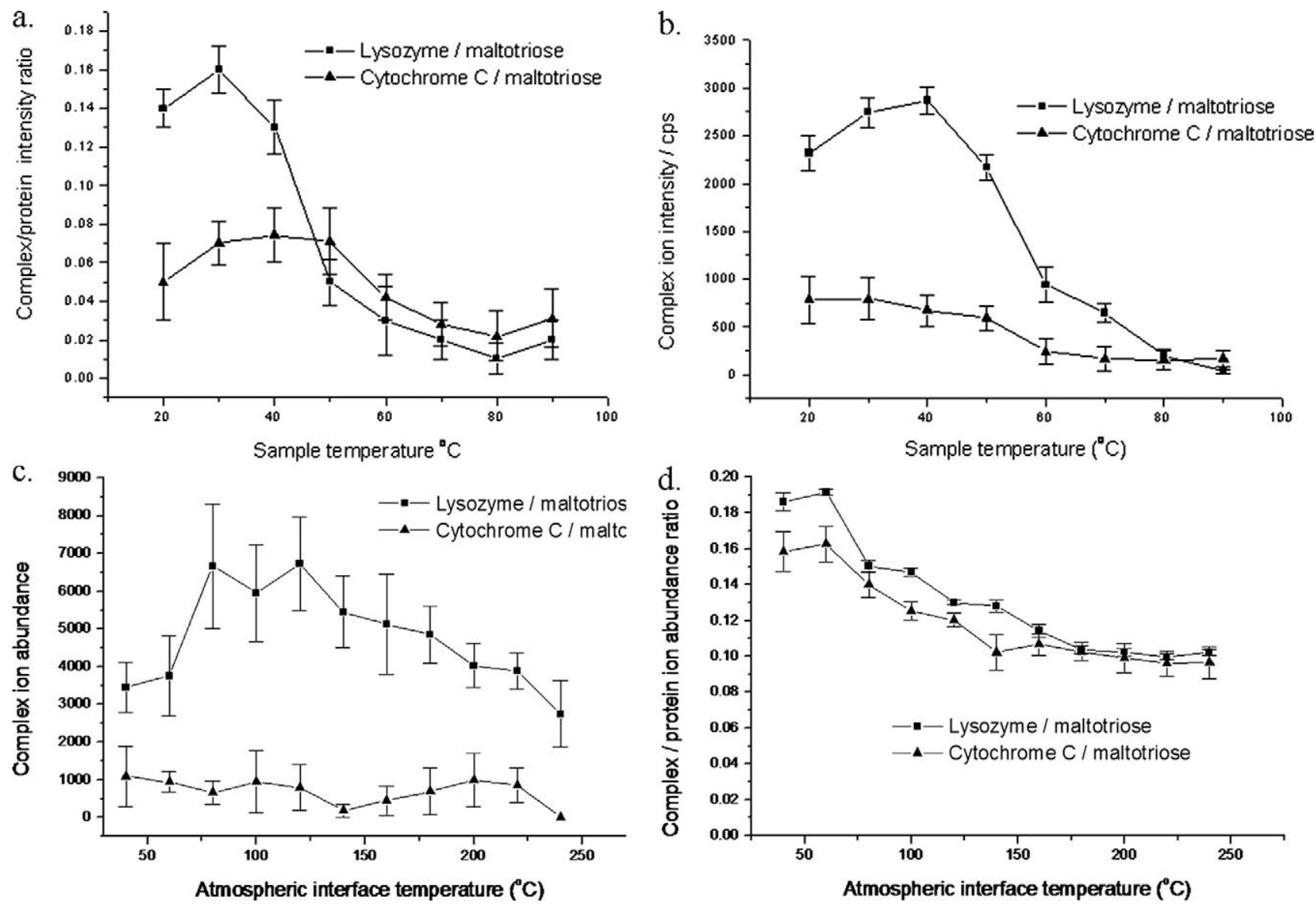

Figure 4. Temperature dependence. (a) Solvent temperature dependence of the complex to protein ion signal intensity ratio in single stage MS. (b) Solvent temperature dependence of the absolute intensity of complex ions in the precursor ion scan for specific and nonspecific protein-ligand complexes. (c) Effect of atmospheric interface temperature on the complex to protein ion signal intensity ratios in single stage MS. (d) Effect of atmospheric interface temperature on absolute intensity of complex ions in the precursor ion scan for specific and nonspecific protein-ligand complexes. 
rate does change complex/protein ion abundance ratios. As described previously, there is a continuous transition between pneumatically assisted micro electrospray and electrosonic spray ionization modes, when flow rate of the nebulizing gas is increased [17, 18]. Generally, this transition is reflected in the continuous improvement of desolvation efficiency of multiply charged macromolecular ions in the atmospheric pressure regime of the mass spectrometer. In case of protein-ligand complexes, the relative intensity of complex ions in single stage MS gradually drops with the increasing flow rate of nebulizing gas and reaches a fairly constant value at high flow rates. Similar phenomenon was observed for both specific and nonspecific complexes. In precursor ion scanning mode, however, there is a considerable difference observed between specific and nonspecific complexes. While the intensity of nonspecific complexes tends to drop to almost zero at higher flow rates, the intensity of specific complex ions does not show similar dramatic dependence on nebulizing gas flow rates.

The observed phenomenon can be explained with the acceleration of gaseous ion formation process due to fast solvent evaporation and atomization of bulk liquid phase. In this case, the equilibrium cannot follow the elevation of protein and ligand concentrations in charged droplets, thus only the preformed complex ions are ionized. At lower flow rates, the residence time of protein and ligand molecules is considerably higher, so the system can react to the increasing concentrations yielding more protein/ligand complex ions. Results agree with observations of Jecklin et al. [20], showing the lack of shift in protein-ligand equilibria during ESSI ionization process. Further evidence supporting this assumption lies in the result of the declustering potential dependence experiments performed at various nebulizing flow rates (vide infra).

Atmospheric interface parameters showed dramatic, although expected effects on the survival rate of complex ions in single stage MS mode (Figure 5a). It is known that most of the protein-ligand complex ion population is lost via dissociation in the ion source and the atmospheric interface of mass spectrometers. Experimental evidences include the 2 to 3 orders of magnitude difference between $\mathrm{K}_{\mathrm{a}}$ values measured by solution phase methods and the fact that these values strongly depend on the employed atmospheric interface a.

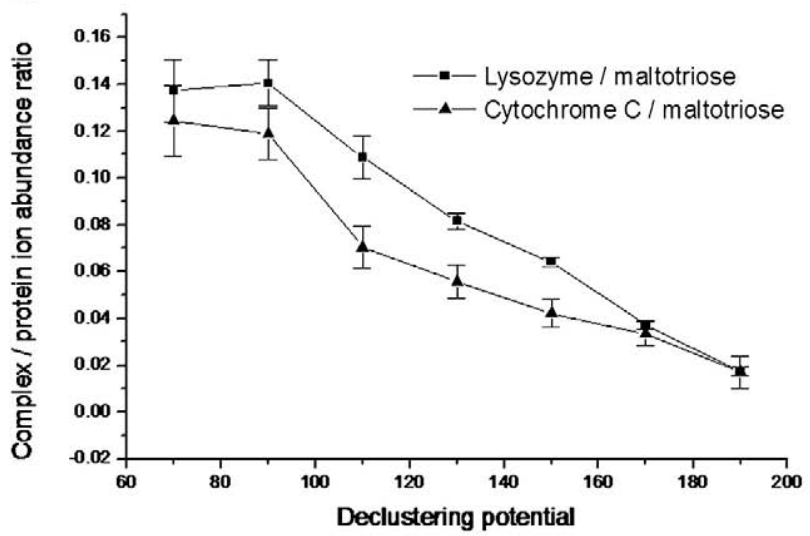

c.

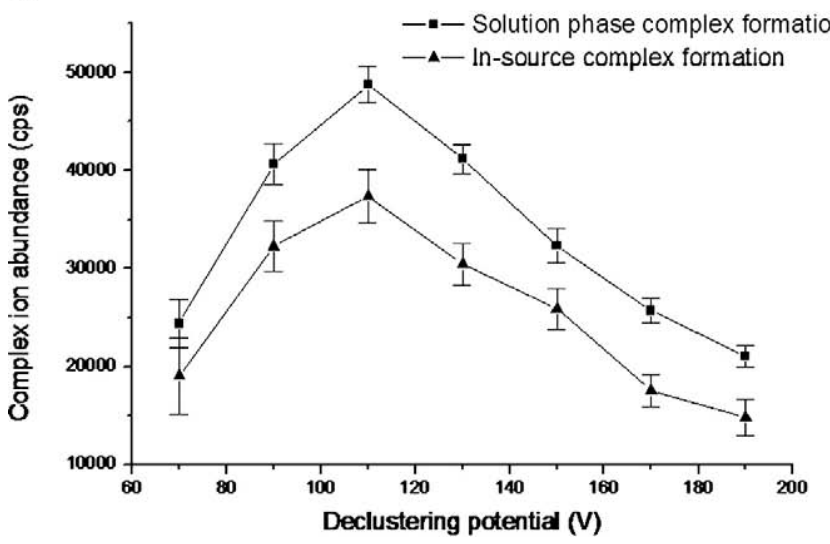

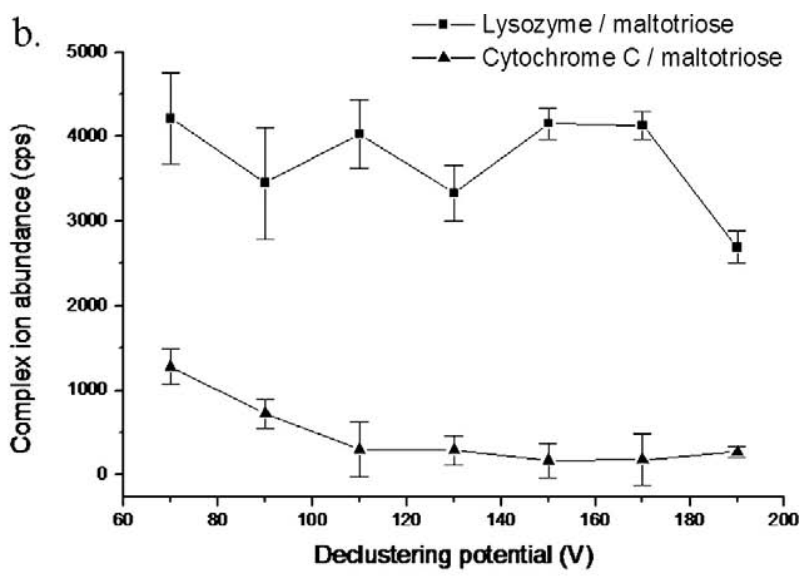

d.

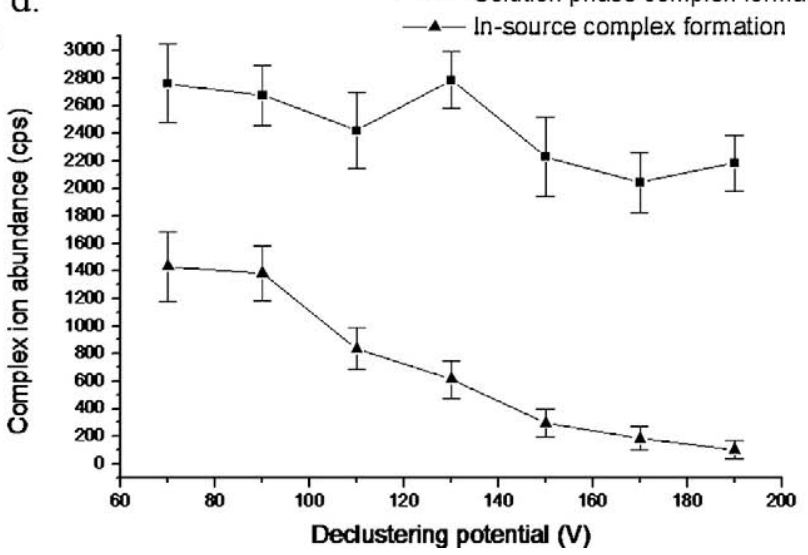

Figure 5. Effect of declustering potential on (a) complex to protein ion signal intensity ratios in single stage MS. (b) Absolute intensity of complex ions in the precursor ion scan for specific and aspecific protein-ligand complexes. (c) Complex ion signal intensity in single stage MS in dual spray experiments. (d) Absolute intensity of complex ions in the precursor ion scan for specific and aspecific protein-ligand complexes in dual spray experiments. 
geometry. Declustering potential is applied between the orifice plate and the skimmer electrode, and its main function is to provide gaseous ions with sufficiently high internal energy to hinder cluster formation in the free jet expansion regime. Hence, increase of declustering potential results in further dissociation of proteinligand complex ions. As it is shown in Figure $5 a$ and $b$, specific and nonspecific complexes follow a similar, linear drop in relative intensity. In contrast to single stage MS data (Figure 5a), precursor ion scan data shows considerable difference between specific and nonspecific complexes (Figure 5b). The ratio of those complexes, which give charged ligand species on dissociation, increases with increasing declustering potential, according to data shown in Figure $5 \mathrm{~b}$. This result has at least two important implications; from a practical point of view it enables the unequivocal differentiation of specific and nonspecific complexes, while from a fundamental point of view it strongly suggests that so-called nonspecific complexes feature different types of interaction between protein and ligand molecules. In this case, the ligand molecules probably do not have access to charged sites of proteins. This type of interaction can be associated with the gas-phase origin of these structures, since nascent multiply charged protein ions tend to "hide" charge sites by self-solvation in the gas phase [33-36].

Interface heater temperature shows similar, though considerably less spectacular, effect both in single stage MS mode and in precursor ion mode (Figure 4c and d). Limited dependence of spectral features from atmospheric interface temperature in case of ESS ionization has been observed previously [17]. The phenomenon was attributed to the fact that ESSI produces fully desolvated ions already in the atmospheric regime of the ion-source/atmospheric interface. Thermal energy provided by the atmospheric interface is sufficiently high to cause dissociation of complexes in solution phase (i.e., in electrosprayed droplets), but it is not enough to induce thermal dissociation of fully desolvated, multiple cation bound complexes.

Ligand concentration is obviously the most important factor having influence on detected protein/complex ion ratios. The ratio is derived as a complex function of the solvent phase equilibrium constant $\left(\mathrm{K}_{\mathrm{a}}\right)$, and the initial protein and ligand concentration $\left([\mathrm{P}]_{0}\right.$ and $[\mathrm{L}]_{0}$, respectively) [22].

Protein-ligand complex formation is described by

$$
K_{a}=\frac{[P L]}{[P][L]}
$$

Original protein and ligand concentration can be given as

$$
[L]_{0}=[L]+[P L]
$$

and

$$
[P]_{0}=[P]+[P L]
$$

Assuming that $[\mathrm{PL}] /[\mathrm{P}]=\mathrm{I}_{\mathrm{PL}} / \mathrm{Is}_{\mathrm{P}}$ for a given charge state, calculated ratio is

$$
R=\frac{[P L]}{[P]}
$$

Substituting eqs 2,3 , and 4 into eq $1, K_{a}$ is

$$
K_{a}=\frac{R^{2}+R}{[L]_{0}(R+1)-[P]_{0} R}
$$

Solving the equation for $\mathrm{R}$, dependence of complex/ protein intensity ratio as function of initial ligand and protein concentration can be given as:

$$
\begin{aligned}
R= & \frac{1}{2}\left(K_{a}[L]_{0}-K_{a}[P]_{0}-1\right. \\
& \left.+\sqrt{\left(K_{a}[L]_{0}-K_{a}[P]_{0}-1\right)^{2}+4 K_{a}[L]_{0}}\right)
\end{aligned}
$$

Although this rather complex formula precisely describes the entire titration curve, for certain segments a simplified expression can be used. If $[\mathrm{L}]_{0} \gg[\mathrm{PL}]$, then $\mathrm{K}_{\mathrm{a}}$ can be approximated as $\mathrm{K}_{\mathrm{a}} \approx[\mathrm{PL}] /[\mathrm{P}][\mathrm{L}]_{0}$, so $[\mathrm{PL}] /[\mathrm{P}] \approx \mathrm{K}_{\mathrm{a}}[\mathrm{L}]_{0}$. Assuming that response factors are similar for protein and complex, and that free ligand concentration is larger than concentration of complex, equation is simplified to: $\mathrm{K}_{\mathrm{a}}[\mathrm{L}]_{0}=\mathrm{I}_{\text {complex }} /$ $\mathrm{I}_{\text {protein. }}$. An important precondition for the assumption made above is the similar charge state distribution for complex and protein, which is generally fulfilled in the studied cases, since ESSI mostly gives spectra featuring predominantly single charge state of protein ions. This means that at initial ligand concentrations high enough to fulfill $[\mathrm{L}]_{0} \gg[\mathrm{PL}]$ the function is linear with slope of $K_{a}$ as it is shown in Figure 6. Hence, the slope of the fitted linear function gives a virtual $\mathrm{K}_{\mathrm{a}}$ value for the system. Since these values are different from literature values (obtained by solution phase methods) by orders of magnitude, they can only be employed to compare stability of different systems. The shape of the $I_{\text {complex }} / I_{\text {protein }}$ versus $[L]_{0}$ curve at even higher concentrations gives an explanation for the difference between $K_{a}$ values obtained by MS and solution phase methods and also for the difference between $\mathrm{K}_{\mathrm{a}}$ values determined by different mass spectrometers. In theory, the curve should continue linearly, until protein signal becomes undetectable, i.e., the curve should approach $\mathrm{I}_{\text {complex, max }} /$

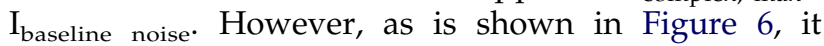
approaches a considerably lower value; then, at even higher concentrations gradually decreases. Thus, the curve can be divided into three sections, as shown in Figure 6 . In the first section it follows theory, and $\mathrm{K}_{\mathrm{a}}$ can be calculated. In the second section $\mathrm{I}_{\text {complex }} / \mathrm{I}_{\text {protein }}$ becomes a constant, independent value from $[\mathrm{L}]_{0}$, and in 

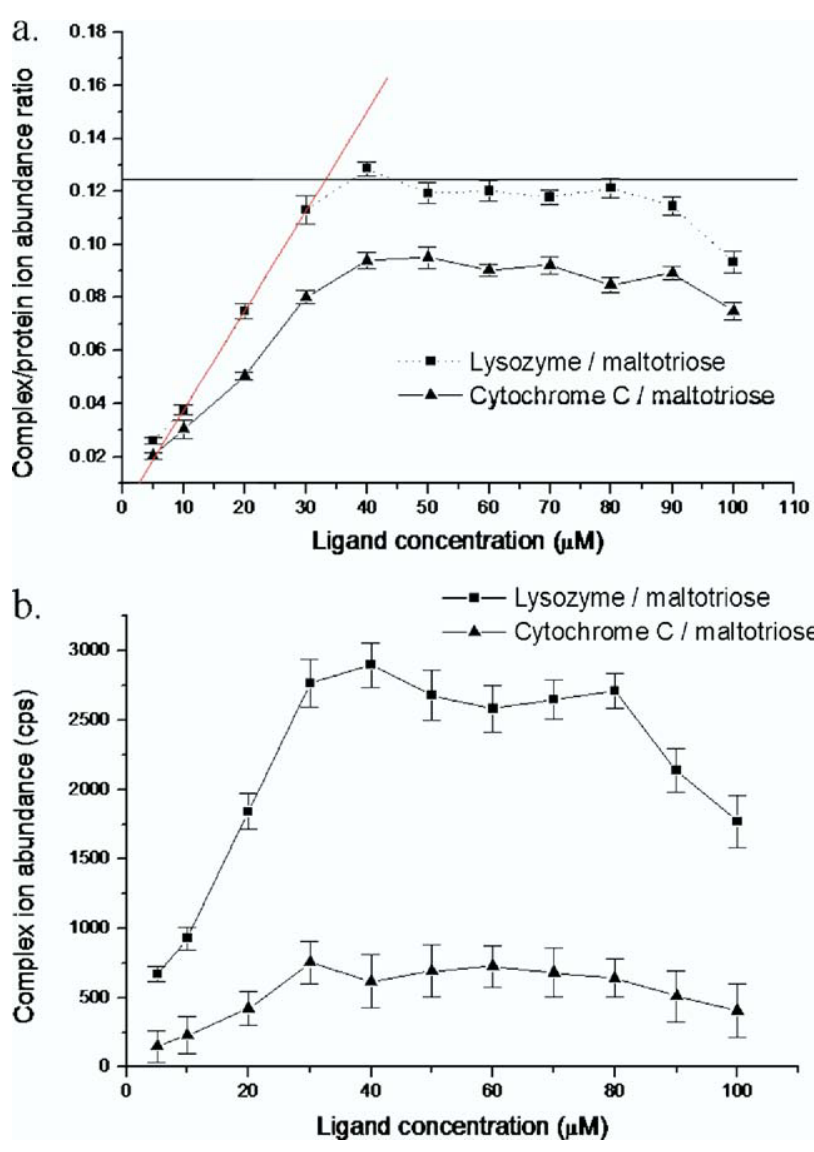

Figure 6. Effect of ligand concentration on (a) complex to protein ion signal intensity ratios in single stage MS. (b) Absolute intensity of complex ions in the precursor ion scan for specific and nonspecific protein-ligand complexes. Red line illustrates maximum measurable complex-to-protein ratio, which is associated with survival ratio of complex ions in the mass spectrometer. For further details, see text.

the third section it gradually decreases with increasing $[\mathrm{L}]_{0}$. The value of $\mathrm{I}_{\text {complex }} / \mathrm{I}_{\text {protein }}$ in the second regime can be associated with the net survival rate of complex ions in the atmospheric interface. Based on solution phase data, at these ligand concentration values, more than $99 \%$ of protein molecules form a complex, so the observed $\mathrm{I}_{\text {complex }} / \mathrm{I}_{\text {protein }}$ ratio is the result of complex dissociation. Since the internal energy distribution of the ion population is different in the case of different atmospheric interfaces/mass spectrometers, this $\mathrm{I}_{\text {com }^{-}}$ plex $/ \mathrm{I}_{\text {protein }}$ value and this virtual $\mathrm{K}_{\mathrm{a}}$ value is also different. The decrease of the ratio in the third regime was tentatively associated with the formation of complexes containing more than one ligand molecule.

The intensity of the complex versus ligand concentration in precursor ion mode follows a similar tendency, as it is shown in Figure 6b. Although nonspecific complex formation gives similar dependence on ligand concentration, the difference between actual values is striking in precursor ion mode. Data strongly suggests that nonspecific complexes do not dissociate the same way as specific complexes do, i.e., fragmentation of these complexes does not yield considerable amount of charged ligand molecules. According to the data, the optimal working range of the precursor ion scan method is the second regime, where intensity of complex ion signal is practically independent of ligand concentration.

Results of the parameter optimization experiments strongly suggested that specific and nonspecific proteinligand complexes differ not only in the biochemical interaction of molecules but at the level of physicochemical interaction as well. To provide evidence for the existence of two types of complexes, preparation of specific and nonspecific complexes were attempted using identical building blocks. A two-spray experiment was designed, where one electrosonic ion source was used to produce gaseous ions of lysozyme, while an orthogonal sonic spray source produced aerosol of maltotriose. The experimental setup is depicted in Figure 1c. Spectra produced by this setup featured complexes of lysozyme with the ligand at various stoichiometries. The experimental setup was also used in a way where lysozyme/maltotriose mixture was sprayed in the ESSI part and pure water in the sonic spray part, which also yielded complexes. Precursor ion scans for the ligand were acquired in both cases at various declustering potential settings. Figures $5 \mathrm{c}$ and $\mathrm{d}$ show the results of the experiment. Dependence of complex ion intensities on declustering potential is comparable in the two sets of experiments in single stage MS, but precursor ion data reveals that the detected complexes show markedly different fragmentation behavior. Complexes formed in solution phase yield considerable amount of ligand ions upon fragmentation, while complexes formed during ionization do not produce ligand ions under similar fragmentation conditions. Ratios of competitive fragmentation processes of ion-bound complexes are determined by the relative ion affinities of the two species, as it is described by the kinetic method. Previous studies concluded that the kinetic method can be successfully applied for multiply charged protein/ligand systems, and discrete proton affinity values can be determined for different charge sites of a protein like lysozyme or cytochrome $c$ [37]. However, the presently described data provides evidence that at least in case of one type of complexes, the kinetic method does not apply and fragment ion ratios are not determined by relative proton affinities of protein ions and ligand molecules. This strongly suggests that at least in one case, ligands do not form ion-bound complex with protein ions.

These results provide direct evidence for the existence of isomeric protein-ligand complexes, even in the case of specifically binding ligands. The data also suggests that nonspecific complexes are not present in liquid phase, but are formed during the gas-phase ion formation process, which is in agreement with biochemical observations.

\section{Conclusions}

Detection of protein complexes in precursor ion scanning mode offers a new, more specific mass spectrometric 
method for the selective detection of biologically relevant protein-ligand complexes. Protein-ligand complex ions are generated by ESSI ionization, and complexes are selectively detected by performing precursor ion scanning for singly charged protonated (or alkali metal adduct) ions of the ligand molecule. While generally applied MS-based methods suffer from nonspecific complex formation during electrospray or MALDI ionization, the methodology demonstrated here minimizes this problem by employing ESSI ionization and precursor ion scan detection. A key observation of the present study was the different fragmentation mechanism of specific and nonspecific protein-ligand complexes. Experiments revealed that there are two types of protein-ligand complex ions formed during spray (ESI or ESSI) ionization. One type was tentatively associated with complexes originally present in solution (specific complexes) and the other type was demonstrated to be formed during ionization. While single stage MS was unable to differentiate them, they can be separated by tandem mass spectrometry, based on their different fragmentation characteristics.

This novel method can be the basis of a new screening technique for the tentative identification of protein targets in the case of compounds with known biological effect, but unknown mechanism of action.

\section{References}

1. Cooks, R. G.; Kruger, T. L. Intrinsic Basicity Determination Using Metastable Ions. J. Am. Chem. Soc. 1977, 99, 1279-1281.

2. Fenn, J. B.; Mann, M.; Meng, C. K.; Wong, S. F.; Whitehouse, C. M. Electrospray Ionization-Principles and Practice. Mass Spectrom. Rev. $1990,9,37-70$.

3. Ganem, B.; Li, Y. T.; Henion, J. D. Detection of Noncovalent Receptor Ligand Complexes by Mass Spectrometry. J. Am. Chem. Soc. 1991, 113, 6294-6296.

4. Hofstadler, S. A.; Sannes-Lowery, K. A. Applications of ESI-MS in Drug Discovery: Interrogation of Noncovalent Complexes. Nat. Rev. Drug Discov. 2006, 5, 585-595.

5. Loo, J. A. Studying Noncovalent Protein Complexes by Electrospray Ionization Mass Spectrometry. Mass Spectrom. Rev. 1997, 16, 1-23.

6. Loo, J. A. Electrospray Ionization Mass Spectrometry: A Technology for Studying Noncovalent Macromolecular Complexes. Int. J. Mass Spectrom. 2000, 200, 175-186.

7. Schlosser, G.; Pocsfalvi, G.; Malorni, A.; Puerta, A.; de Frutos, M.; Vékey, K. Detection of Immune Complexes by Matrix-Assisted Laser Desorption/Ionization Mass Spectrometry. Rapid Commun. Mass Spectrom. 2003, 17, 2741-2747.

8. Woods, A. S.; Huestis, M. A. A Study of Peptide-Peptide Interaction by Matrix-Assisted Laser Desorption/Ionization. J. Am. Soc. Mass Spectrom. 2001, 12, 88-96.

9. Farmer, T. B.; Caprioli, R. M. Determination of Protein-Protein Interactions by Matrix-Assisted Laser Desorption/Ionization Mass Spectrometry. J. Mass Spectrom. 1998, 33, 697-704.

10. Wortmann, A.; Pimenova, T.; Alves, S.; Zenobi, R. Investigation of the First Shot Phenomenon in MALDI Mass Spectrometry in Protein Complexes. Analyst 2007, 132, 199-207.

11. Salih, B.; Zenobi, R. MALDI Mass Spectrometry of Dye-Peptide and Dye-Protein Complexes. Anal. Chem. 1998, 70, 1536-1543.

12. Li, Y.; Shrestha, B.; Vertes, A. Atmospheric Pressure Molecular Imaging by Infrared MALDI Mass Spectrometry. Anal. Chem. 2007, 79, 523-532.

13. Hernández, H.; Robinson, C. V. Determining the Stoichiometry and Interactions of Macromolecular Assemblies from Mass Spectrometry. Nat. Protoc. 2007, 2, 715-726.

14. Hart, C. P. Finding the Target after Screening the Phenotype. Drug Discov. Today 2005, 10, 513-519.

15. Oravcova, J.; Bohs, B.; Lindner, W. Drug-Protein Binding Sites. New Trends in Analytical and Experimental Methodology. J. Chromatogr. B Biomed. Appl. 1996, 667, 1-28.
16. van den Heuvel, R. H.; van Duijn, E.; Mazon, H.; Synowsky, S. A.; Lorenzen, K.: Versluis, C.; Brouns, S. J.; angridge, D.; van der Oost, J. Hoyes, J.; Heck, A. J. Improving the Performance of a Quadrupole Time-of-Flight Instrument for Macromolecular Mass Spectrometry. Anal. Chem. 2006, 78, 7473-7483.

17. Takats, Z.; Wiseman, J. M.; Gologan, B.; Cooks, R. G. Electrosonic Spray Ionization. A Gentle Technique for Generating Folded Proteins and Protein Complexes in the Gas Phase and for Studying Ion-Molecule Reactions at Atmospheric Pressure. Anal. Chem. 2004, 76, 4050-4058.

18. Wiseman, J. M.; Takats, Z.; Gologan, B.; Davisson, V. J.; Cooks, R. G. Direct Characterization of Enzyme-Substrate Complexes by Using Electrosonic Spray Ionization Mass Spectrometry. Angew. Chem. Int. Ed. 2005, 44, 913-916.

19. Nesatyy, V. J. Mass Spectrometry Evaluation of the Solution and Gas-Phase Binding Properties of Noncovalent Protein Complexes. Int. J. Mass Spectrom. 2002, 221, 147-161.

20. Jecklin, M. C.; Touboul, D.; Wortmann, A.; Bovet, C.; Zenobi, R. Which Electrospray-Based Ionization Method Best Reflects Protein-Ligand Interactions Found in Solution? A Comparison of ESI, nanoESI, and ESSI for the Determination of Dissociation Constants with Mass Spectrometry. J. Am. Soc. Mass Spectrom. 2008, 19, 332-343.

21. Kebarle, P. A Brief Overview of the Present Status of the Mechanisms Involved in Electrospray Mass Spectrometry. J. Mass Spectrom. 2000, 35, 804-817.

22. Daniel, J. M.; McCombie, G.; Wendt, S.; Zenobi, R. Mass Spectrometric Determination of Association Constants of Adenylate Kinase with Two Noncovalent Inhibitors. J. Am. Soc. Mass Spectrom. 2003, 14, 442-448.

23. Cheng, X. H.; Chen, R.; Bruce, J. E.; Schwartz, B. L.; Anderson, G. A. Hofstadler, S. A.; Gale, D. C.; Smith, R. D.; Gao, J.; Sigal, G. B.; Mammen, M.; Whitesides, G. M. Using Electrospray-Ionization FTICR MassSpectrometry to Study Competitive-Binding of Inhibitors to CarbonicAnhydrase. I. Am. Chem. Soc. 1995, 117, 8859-8860.

24. Gao, J. M.; Cheng, X.; Chen, R.; Sigal, G. B.; Bruce, J. E.; Schwartz, B. L.; Hofstadler, S. A.; Anderson, G. A.; Smith, R. D.; Whitesides, G. M. Screening Derivatized Peptide Libraries for Tight Binding Inhibitors to Carbonic Anhydrase II by Electrospray Ionization Mass Spectrometry. J. Med. Chem. 1996, 39, 1949-1955.

25. Gao, J. M.; Carbeck, J.; Lei, Q. P.; Smith, R. D.; M., W. G. Probing the Energetics of Dissociation of Carbonic Anhydrase-Ligand Complexes in the Gas Phase. Biophys. J. 1999, 76, 3253-3260.

26. Wigger, M.; Eyler, J. R.; Benner, S. A.; Li, W.; Marshall, A. G. Fourier Transform-Ion Cyclotron Resonance Mass Spectrometric Resolution, Identification, and Screening of Noncovalent Complexes of Hck Src Homology 2 Domain Receptor and Ligands from a 324-Member Peptide Combinatorial Library. J. Am. Soc. Mass Spectrom. 2002, 13, 1162-1169.

27. Annis, D. A.; Shipps, G. W. J.; Deng, Y.; Popovici-Müller, I.; Siddiqui, M. A.; Curran, P. J.; Gowen, M.; Windsor, W. T. Method for Quantitative Protein-Ligand Affinity Measurements in Compound Mixtures. Anal. Chem. 2007, 79, 4538-4542.

28. Slon-Usakiewicz, J. J.; Ng, W · Dai, J. R. Pasternak, A · Redden, P. R. Frontal Affinity Chromatography with MS Detection (FAC-MS) in Drug Discovery. Drug Discov. Today 2005, 10, 409-416.

29. Becker, R. R.; Halbrook, J. L.; Hirs, C. H. W. Isolation and Characterization of Ovine Ribonuclease A, B, and C from Pancreatic Secretion. J. Biol. Chem. 1973, 248, 7826-7832.

30. Wilm, M. S.; Mann, M. Electrospray and Taylor-Cone Theory, Doles Beam of Macromolecules at Last. Int. J. Mass Spectro. Ion Processes. 1994, $136,167-180$

31. Wilm, M.; Mann, M. Analytical Properties of the Nanoelectrospray Ion Source. Anal. Chem. 1996, 68, 1-8.

32. Schmidt, A.; Karas, M. The Influence of Electrostatic Interactions on the Detection of Heme-Globin Complexes in ESI-MS. J. Am. Soc. Mass Spectrom. 2001, 12, 1092-1098.

33. McLafferty, F. W.; Guan, Z.; Haupts, U.; Wood, T. D.; Kelleher, N. L. Gaseous Conformational Structures of Cytochrome c. J. Am. Chem. Soc. 1998, 120, 4732-4740.

34. Horn, D. M.; Breuker, K.; Frank, A. J.; McLafferty, F. W. Kinetic Intermediates in the Folding of Gaseous Protein Ions Characterized by Electron Capture Dissociation Mass Spectrometry. J. Am. Chem. Soc. 2001, 123, 9792-9799.

35. Badman, E. R.; Hoaglund-Hyzer, C. S.; Clemmer, D. E. Monitoring Structural Changes of Proteins in an Ion Trap Over Similar to 10-200 ms: Unfolding Transitions in Cytochrome $c$ ions. Anal. Chem. 2001, 73, 6000-6007.

36. Oh, H.; Breuker, K.; Sze, S. K.; Ge, Y.; Carpenter, B. K.; McLafferty, F. W. Secondary and Tertiary Structures of Gaseous Protein Ions Characterized by Electron Capture Dissociation Mass Spectrometry and Photofragment Spectroscopy. Proc. Nat. Acad. Sci. U.S.A. 2002, 99, 15863-15868.

37. Schnier, P. D.; Gross, D. S.; Williams, E. R. On the Maximum ChargeState and Proton-Transfer Reactivity of Peptide and Protein Ions Formed by Electrospray-Ionization. J. Am. Soc. Mass Spectrom. 1995, 6 , 1086-1097. 